\title{
Harmonious colors: from alchemy to science
}

\section{Giordano Beretta, Nathan Moroney}

Giordano B. Beretta, Nathan M. Moroney, "Harmonious colors: from alchemy to science," Proc. SPIE 8292, Color Imaging XVII: Displaying, Processing, Hardcopy, and Applications, 829201 (24 January 2012); doi: 10.1117/12.915839

Event: IS\&T/SPIE Electronic Imaging, 2012, Burlingame, California, United States 


\title{
Harmonious colors: from alchemy to science
}

\author{
Giordano B. Beretta, Nathan M. Moroney \\ Hewlett-Packard Laboratories, Printing and Content Delivery Lab \\ 1501 Page Mill Road, Palo Alto, USA
}

\begin{abstract}
There is a very long tradition in designing color palettes for various applications, going back to at least the Upanishad. Although color palettes have been influenced by the available colorants, starting with the advent of aniline dyes in the late 1850 s there have been few physical limits on the choice of individual colors. This abundance of choices exacerbates the problem of limiting the number of colors in a palette, i.e., in keeping them into a manageable quantity.

For example, it is not practical for a car company to offer each model in hundreds of colors. Instead, for each model year a small number of color palettes is offered, each containing the colors for the body, trim, interior, etc. Another example is the fashion industry, where in addition to solid colors there are also patterns, leading to a huge variety of combinations that would be impossible to stock.

The traditional solution is that of "color forecasting." Color consultants assess the sentiment or affective state of a target customer class and compare it with new colorants offered by the industry. They assemble a limited color palette, name the colors according to the sentiment, and publish their result. Textile manufacturers will produce fabrics in these colors and fashion designers will design clothes, accessories, and furniture based on these fabrics. Eventually, the media will communicate these forecasts to the consumers, who will be admired by their cohorts when they choose colors from the forecast palette, which by then is widely diffused.

The color forecasting business is very labor intensive and difficult, thus for years computer engineers have tried to come up with algorithms to design harmonious color palettes, alas with little commercial success. For example, Johannes Itten's color theory has been implemented many times, but despite Itten's success in the Bauhaus artifacts, the computer tools have been of little utility. Indeed, contrary to the auditory sense, there is no known physiological mechanism sustaining harmony and the term "harmonious" just has the informal meaning of "going well together."

We argue that the intellectual flaw resides in the belief that a masterful individual can devise a "perfect methodology" that the engineer can then reduce to practice in a computer program. We suggest that the correct approach is to consider color forecasting as an act of distillation, where a palette is digested from the sentiment of a very large number of people. We describe how this approach can be reduced to an algorithm by replacing the subjective process with a data analytic process.
\end{abstract}

Keywords: color science; color palettes; color harmony; crowdsourcing

\section{INTRODUCTION}

When dealing with color, people rarely deal with a single color in isolation. Most of the time, they deal with a palette of colors, also called color scheme or color template. The colors in a palette are seldom haphazard, usually they have to fulfill some constraint like being in harmony or contrasting. For example, when we paint our flat, we would like our wall colors to harmonize and the trim to contrast with the walls. ${ }^{1}$

In the case of paints, the problem is that we have to chose from thousands of different colors. In digital applications the problem is even worse, because a typical display can address millions of colors. Ideally palettes should be small, with about a dozen colors.

Further author information: (Send correspondence to N.M.M.)

G.B.B.: E-mail: giordano.beretta@hp.com, Telephone: +1 6508576713

N.M.M.: E-mail: nathan.moroney @ hp.com, Telephone: +1 6502362825

Blog: mostlycolor.ch

Color Imaging XVII: Displaying, Processing, Hardcopy, and Applications, edited by Reiner Eschbach, Gabriel G. Marcu, Alessandro Rizzi, Proc. of SPIE-IS\&T Electronic Imaging, SPIE Vol. 8292, 82920I - (c) 2012 SPIE-IS\&T · CCC code: 0277-786X/12/\$18 · doi: 10.1117/12.915839 
It is even very difficult to chose a first starting (or key) color. In the case of a set of slides for a presentation one might start for example with the main color in the corporate logo, but sometimes it may not be in tone with the theme of the presentation, like a presentation on green technology by a representative from a company with a red logo.

Adding other colors to a palette is even harder. A simple task like choosing a contrasting text color for a given background is difficult, because for example on the above green background, a red text would not be very readable and not readable at all for a person with color vision deficiency; ${ }^{2-6}$ this is true also for logos. ${ }^{7}$

A more complex task is that of adding harmonious colors, because there is no physical nor physiological basis for color harmony. Furthermore, as we hinted in the case of green technology, people may want to express a sentiment with a color palette. The relation between color palettes and sentiments is highly dependent on culture, subculture, social economic status, geography, and time.

We propose a cloud service that can be used from any mobile or stationary device. The user can describe the constraints for a palette (sentiment, application, event, etc.) and the service will propose a small number $\left(7 \pm 2\right.$, see $\left.\operatorname{Miller}^{8}\right)$ of harmonious color palettes.

\section{HISTORICAL PERSPECTIVE}

In the western or Judeo-Christian culture, we have the notion of the philosopher or sage who can acquire omniscience and guide those in the dark through the acquisition of the lapis philosophorum (philosopher's stone). One of the fallacies of this approach is that it can quickly lead to esoteric cosmological generalizations like those found in the Kabbalah. Numerology is a frequently used technique, along with astrology and alchemy.

Although these disciplines have been largely replaced by mathematics, astronomy, respectively chemistry in modern science, people not formally trained in modern science can easily fall back into the old disciplines, because they so are so well ingrained in our western culture. For example, Johannes Itten — a professor at the Zürich University of the Artsdeveloped a Fablehre or color theory based on the color circle shown on the left in Fig. 1. The circle on the right is taken from the original student edition ${ }^{9}$ of the book and reveals how the circle is constructed, namely by color terms and not by perceptual attributes. In Itten's theory, two diametrically opposite colors are called direct complements, while the colors on opposite vertices of a circumscribed equilateral triangle are called triadic complements. The colors adjacent to a given color are called harmonious colors.
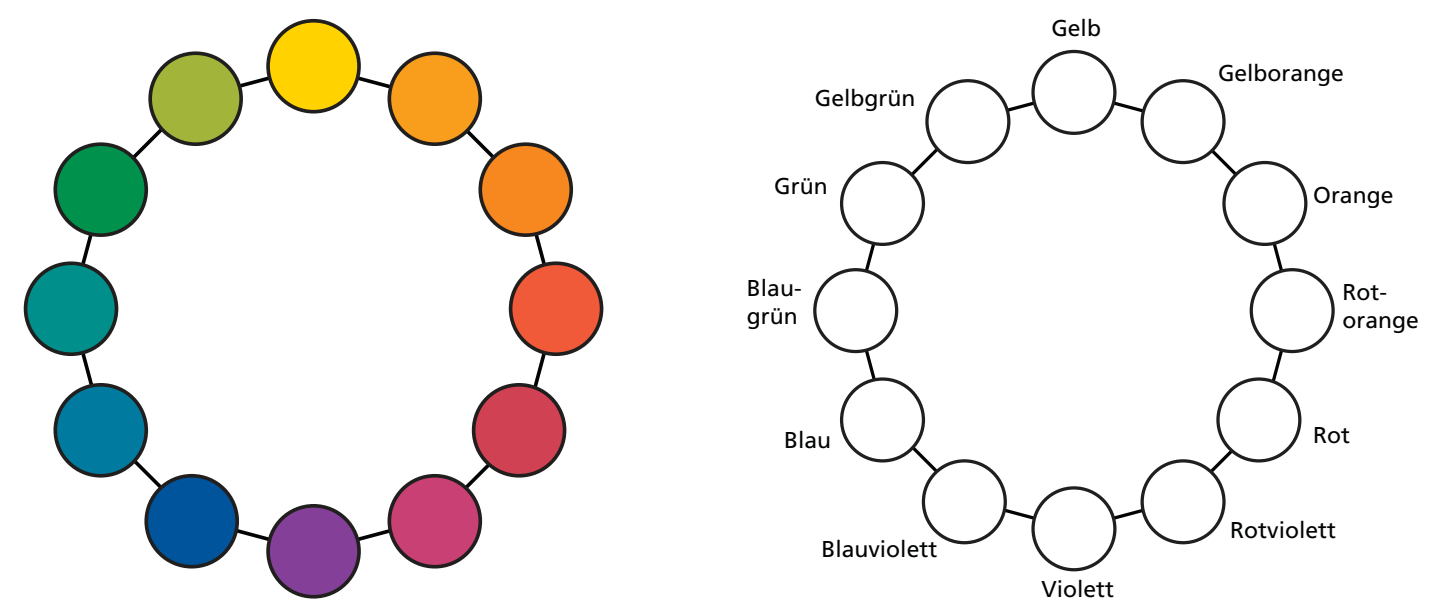

Figure 1. Left: A colored rendering of Itten's Farbkreis based on the NCS color atlas. Right: The student edition of Itten's book reveals how the color cirlce is constructed, viz. by placing six monolexemic primary colors on a circle; in a second step a bilexemic secondary color is placed between each consecutive pair of primary colors.

It is relatively straightforward to create a color selection tool blending opponent color theory with Itten's Farblehre. ${ }^{10-15}$ However, as soon as the tool in these references was deployed, its limited practical value was immediately exposed. The color theory is an excellent tool to teach color, but is not suitable for selecting colors because it is too regimented. Based 
on his experience, Itten created a theory that is useful to promulgate his knowledge, but not to create a practical tool. Fortunately, we had concomitantly developed a different color selection tool described in the same paper and video, but a different patent application. ${ }^{16}$

Originally, we envisaged the two tools for completely different purposes. The tool based on color theory (Proto-Palette) was supposed to help selecting color palettes for functional color, like descriptive statistics charts and diagrams. The second tool (Digital Palette) was essentially a database of color palettes taken from the physical environment. The culturally based palettes were constructed by researching the natural colorants available to a culture or region before the advent of aniline dyes. The artistic palettes where obtained by having an art painter reconstruct the palette of each artist and then measuring these palettes with a spectrophotometer.

When the two tools were deployed in 1989 to design the desktop of a new computer system, the graphic designers used mostly the reference color selection system and its color blending function. They only rarely used the tool based on color theory, and then only to add regimented colors to a palette in the database and tweaking them with a third tool called Meta-Palette.

At the time, this experience made us realize that despite our knowledge of color science, we could not develop a simple tool for generating harmonious palettes based on symmetries in a perceptually uniform color space. We thought what was more important were archetypes, patterns engrained in a culture's collective mind. Today, after 20 years we are ready to take this insight to the next level.

As western culture is suffering from the hangover after the Sputnik crisis has been overcome, the lead in scientific study and technological progress has passed to the cultures of the Far East. While in the western cultures we have the concept of the omniscient philosopher, in the Far East they have the antithetical view of the people as a whole, and this is the lead to the next step in designing color palette selection tools, as indicated in Fig. 2.

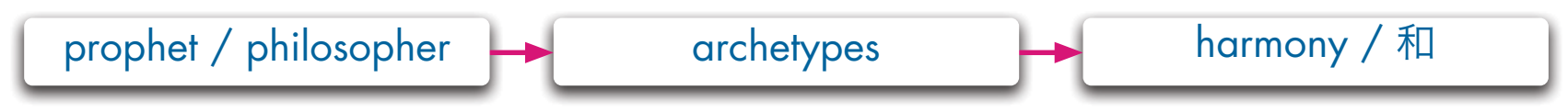

Figure 2. In the western culture we historically had the concept of the omniscient prophet or philosopher. However, we quickly learned that an alchemist's approach to color palette selection does not lead to useful tools. The study of archetypes proved to be a more more fruitful approach, but today we must embrace the far eastern concept of harmony.

The Chinese character in the last box of Fig. 2 has three readings. One is that of harmony in the western sense, but it also means people (and domestic) and it means sum. Incidentally, when this character is preceded by the character for flat, we obtain the word peace. While in the western culture we tried to find the lapis philosophorum or at least to find the philosopher possessing it, our eastern colleagues teach us that we must find the wisdom of harmony in the sum of the people, or crowd to use a trendy term.

In the remainder of this paper we present a methodology to distill pleasing color palettes by sourcing the wisdom of crowds. When we write of harmony, we intend this word both in the western sense of pleasing as well as in its more complex far eastern amalgamated meanings. Interestingly, Far Easterners often like to depict harmony as a circle of children holding their hands, and the Unesco has often used this metaphor in their logos, as shown in an example in Fig. 3 in the center.

\section{COLOR RESEARCH VIA CROWDSOURCING}

While the philosopher can sit in his garage and brood over a cosmology, the scientist must poll the sum of people, or at least a very large crowd. A problem related to the creation of harmonious color palettes is that of color naming, i.e., eliciting the common terms for a color stimulus. Fortunately there is already significant experience in the creation and use of very large lexical color resources specific to displays, building on a decade long effort to collect unconstrained color names on the World Wide Web by Moroney (2003). ${ }^{17}$ Recent results by Mylonas ${ }^{18}$ suggest excellent agreement for two different Web-based color naming experiments.

A further result published on the Web by Munroe and Ellis ${ }^{19}$ has increased the scale of data publicly available for analysis from thousands to millions of participants. This has considerable promise for attaining a detailed understanding of the use of color terms, but has required analysis and thought with respect to a systematic method to validate the data. 

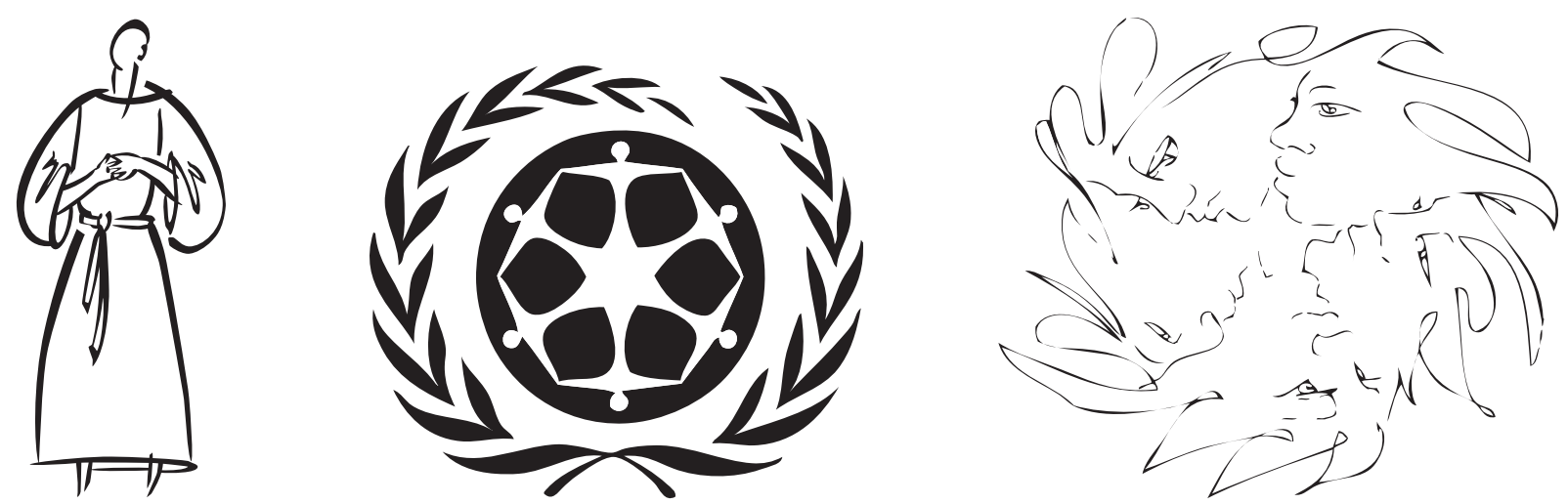

Figure 3. In the western culture the hero is the individual, while in the far east it is the people in harmony, as shown in Unesco's logos of the population world year (id 20004489) and the cultural development decade (id 20006215), (OUnesco.

Due to the data size, manual inspection of individual responses is not an option. In a recent paper ${ }^{20}$ we described how to effectively and efficiently perform a laboratory validation of this large scale uncontrolled Web data, following Zuffi et al. ${ }^{21}$

This is critical given the informal nature of the survey. Munroe and Ellis are not scientists and their tagline is "a webcomic of romance, sarcasm, math, and language," which suggests that their main audience might be young and opinionated. In their survey they displayed color patches and asked their readers to name them. They collected over 3.5 million terms.

We limited ourselves to verifying the basic color terms, yielding 1.3 million terms. In our strictly controlled experiment with 16 screened observers, for each basic color term with displayed the patches for whose color the term was used. Our observers were instructed to validate the data, i.e., to indicate which patches indeed correspond to each color term. Tab. 1 shows that for the basic color terms, $33 \%$ to $74 \%$ of the data could be retained. Given the size of the data corpus, the total numbers are still very large.

\begin{tabular}{cccccccc}
\hline brown & purple & pink & orange & blue & yellow & red & green \\
\hline 33 & 44 & 49 & 49 & 53 & 70 & 73 & 74 \\
\hline
\end{tabular}

Table 1. Percentage of color term data points contributed that were retained after validation.

Moreover, the CIE $1976\left(L^{*} a^{*} b^{*}\right)$ hue correlation with the Web data (complete and validated) to Berlin and Kay 22 is over 0.96 . The correlations for lightness are less but still on the order 0.65 . Finally the hue and lightness correlations for the complete and validated data sets is shown to be 0.99 , suggesting that the scale and limited noise in this case may mean that validation is in fact not necessary (see our AIC 2011 paper $^{20}$ for the details).

In summary, while research related to color vision and the physiology require precisely controlled laboratory experiments, many question related more to color cognition—e.g., readability, color naming, color esthetics—can be studied more reliably though large crowd-sourcing experiments. When the polled population is very large, outliers like persons with defective color vision or disruptive persons cancel out and do not affect the final result.

Donovan, Agarwala, and Hertzmann ${ }^{23}$ have performed big data analytics on large datasets in a first model-free data analysis of crowdsourced color palettes. They studied three datasets: Adobe's Kuler, COLOURLovers, and a more controlled dataset created through Amazon's Mechanical Turk. The emphasis is on model-free, and in this sense we have some reservations on the more controlled dataset. Furthermore, it should be noted that these datasets are associated mostly with professional designers, who typically only need a key color and a contrasting color, while they create additional palette elements through tints and shades. We speculate that non-professional users need half a dozen or a dozen of colors in a palette, for example to color a graph or a pie chart, because they are less versed in specifying variants of a color. Despite these minor reservations, we think their work is very important.

One color cognitive aspect that can be studied through very large corpora is that of ephemerality. In Fig. 4 we used Google's English book corpus and tracked the number times some synonyms of cyan appear in books each year from 
1800 to 2000 . The diagram suggests that to be aligned with fellow English speaking people, we should use the color term turquoise instead of cyan. Of course, in the case of the process ink, we should still use the term cyan.

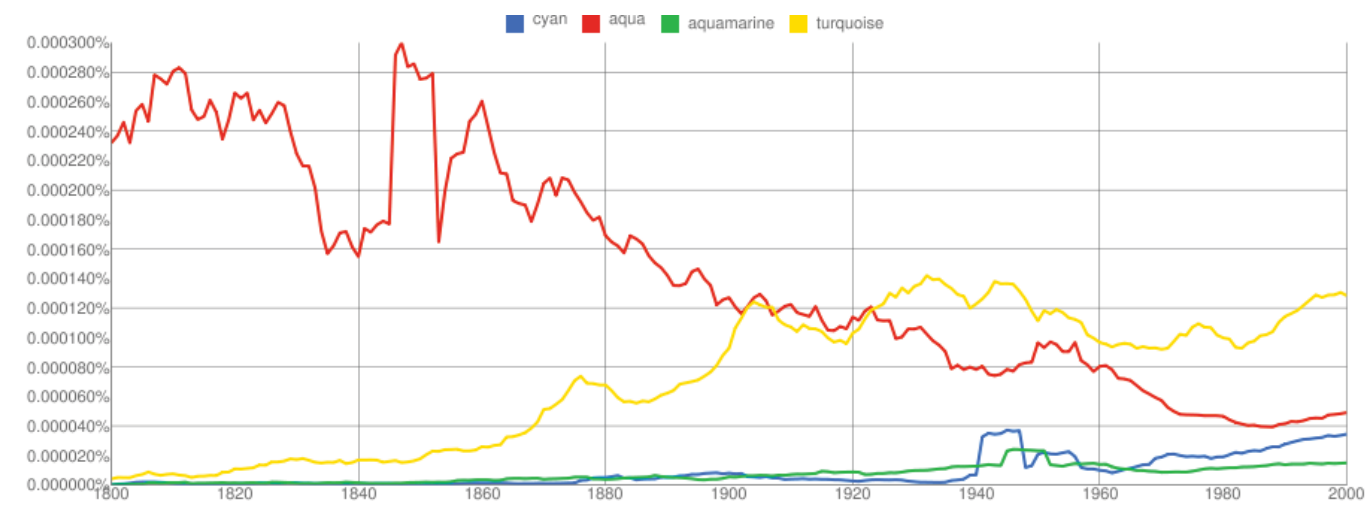

Figure 4. This diagram illustrates how cyan is not the best term for $\operatorname{RGB}=(0,1,1)$ and we should use the term turquoise instead.

We can use the crowd-sourcing approach also to answer very difficult questions, like the number of basic color terms. The diagram in Fig. 5 illustrates that the color between yellow and green might be becoming a basic term also in English like it is in far eastern languages, because chartreuse is monolexemic and is replacing olive green.

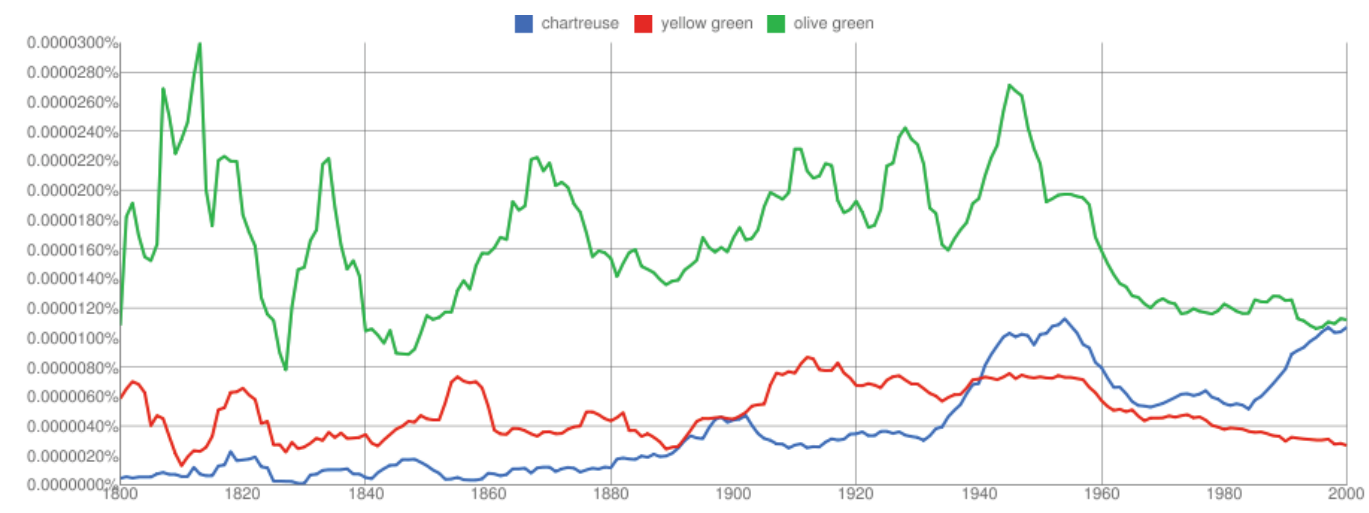

Figure 5. Is the term for RGB $=(1,1,0)$ monolexemic?

\section{A HARMONIOUS METHODOLOGY FOR CREATING HARMONIOUS COLOR PALETTES}

The temporal effect is even more pronounced in the case of color palettes, because colors are typically forecast for every season. At the beginning of the previous section we wrote about the philosopher brooding in the garage. When we consider color forecasters, they operate quite differently, more like filters and meme amplifiers. They have to be very aware of what is happening in the world and have a general feeling of people's collective sentiment. They also have to hierarchically receive input form regional or topical forecasters and integrate it at each level. Croudsourcing allows us to do this algorithmically.

From Canon's Color Advisor ${ }^{24}$ we know that we can successfully classify color palettes by event or by sentiment. Thus the first thought would be to use a semantic knowledge base like Yago2 or Freebase to periodically create a graph database related to color. However, this approach would be based on an ontology, which in turn requires a philosopher for its organization.

The palette database is created in the cloud by periodically crawling the Web for color images. In a first step the images are culled to exclude impertinent images (small images that can be buttons, logos, etc.; portraits, where the sentiment may be referring to the facial expression rather than the colors). 
In a second step, the images are color quantized to reduce the number of colors in them. We propose to quantize the images lexically, ${ }^{25}$ i.e., to assign all pixels whose colors have the same color term, to the same bin. This step reduces the color complexity to a level manageable by linguistic cognitive mechanisms. The resulting set of colors forms the image's color palette. For each color, the number of pixels in that color is also recorded, normalized to the image size.

For the lexical quantization we need a color term corpus. In the past we have used fixed dictionaries proposed by experts, viz. the ISCC dictionary ${ }^{26}$ and the Coloroid dictionary. ${ }^{27}$ Today we prefer dynamic dictionaries obtained through crowdsourcing, because they are larger and are evolving in time; examples are Moroney's color dictionary ${ }^{17}$ and the xkcd corpus. ${ }^{19}$ Similar projects have been carried out by Mylonas, ${ }^{18}$ Tominaga, ${ }^{28}$ and others.

In a third step, the text around the image is scanned and appropriate keywords are harvested. Appropriateness is meant to include such terms as sentiments, events, etc. Other metadata that is easily collected includes the geographical location (from the IP address or GIS tags), the culture (from the set of languages in the http request), etc.

In a fourth step, a database is created that stores the color palettes, the area of each color, and the keywords. The area, i.e., normalized number of pixels of each color, is a very simple measure. Better metrics based on computer vision have been studied by Sandra Skaff et. al. ${ }^{29}$

When a user queries the harmonious color palette cloud service, the database is queried with the specified keywords and the relevant palettes are retrieved. Because of the grey world hypothesis, it is not possible to average the colors returned with the query. Instead the palettes are clustered and for the top $7 \pm 2$ (see Miller ${ }^{8}$ ) clusters, each averaged palette is proposed.

The palette is served to the user's mobile or stationary device from the cloud service. The palette can be presented in the form of a collection of color swatches. On a mobile device with location services available, the colors can also be identified by context. For example, it the user is in a paint store, the names and numbers of the store's brand are displayed. In a furniture store, the items of with colors in the palette are listed.

\section{OUTLOOK}

The idea of creating color palette advisors based on color theories has been proposed many times, but in practice such computer tools are not very useful. We take a long view and propose that instead of a heuristic scheme, a color palette advisor should be dynamic in time and be based on crowdsourcing and big data analytics. We are currently implementing the components of such a system and will report on its usefulness in a future paper.

\section{REFERENCES}

[1] Beretta, G. B. and Nonaka, Y., "House painting with NCS in the USA," Atti della Fondazione Giorgio Ronchi LV(1), 93-118 (2000). http: / /www. inventoland. net/pdf/Reports/iscc2 .pdf.

[2] Beretta, G. B., "Color aspects of variable data proofing," in [Internet Imaging VI], Santini, S., Schettini, R., and Gevers, T., eds., 5670, 175-182, SPIE (January 2005). http://dx. doi.org/10.1117/12.592489.

[3] Beretta, G. B., "Pre-flighting variable data print jobs using the coloroid system," in [The 10th Congress of the International Colour Association ], 2, 1231-1234 (May 2005).

[4] Zuffi, S., Beretta, G. B., and Brambilla, C., "A color selection tool for the readability of textual information on web pages," in [Internet Imaging VII], Santini, S., Schettini, R., and Gevers, T., eds., IS\&T/SPIE Symposium on Electronic Imaging Science \& Technology 6061, 606108, IS\&T/SPIE, SPIE, San Jose (California, USA) (January 2006).

[5] Zuffi, S., Brambilla, C., Beretta, G. B., and Scala, P., "Human computer interaction: Legibility and contrast," in [14th International Conference on Image Analysis and Processing], 241-246, IEEE Computer Society, 10662 Los Vaqueros Circle, PO Box 3014, Los Alamitos, CA 90720-1264 USA (September 10-14 2007).

[6] Zuffi, S., Brambilla, C., Beretta, G. B., and Scala, P., "Understanding the readability of colored text by crowdsourcing on the web," External HPL-2009-182, HP Laboratories (August 6 2009). http: / www.hpl.hp.com/ techreports/2009/HPL-2009-182.html.

[7] Beretta, G. B., "Adaptive color artwork," in [Color Imaging XII: Processing, Hardcopy, and Applications], Eschbach, R. and Marcu, G. G., eds., IS\&T/SPIE Symposium on Electronic Imaging Science \& Technology 6493, 64930L, SPIE (January 2007). 
[8] Miller, G. A., "The magical number seven, plus or minus two: Some limits on our capacity for processing information," Psychological Review 63(2), 81-97 (1956). http:/ / psychclassics.yorku.ca/Miller/.

[9] Itten, J., [Kunst der Farbe (Studienausgabe)], Ravensburger Buchverlag Otto Maier GmbH (1987).

[10] Stone, M. C., [A Field Guide to Digital Color], A K Peters, Natick, MA (July 28 2003). http: / / www . crcpress . $\mathrm{com} / \mathrm{product/isbn/9781568811611.}$

[11] Beretta, G. B., "Color palette selection tools," in [Advanced Printing of Conference Summaries, SPSE's 43rd Annual Conference ], 94-96, The Society for Imaging Science and Technology (May 20-25 1990). http://www . inventoland.net/pdf/Reports/gbb1990paper.pdf.

[12] Beretta, G. B. and Stone, M. C., "Color palette selection tools (video)," in [CHI'90 Technical Video Program—New Techniques], ACM SIGGRAPH Video Review Issue 55 (1990). http: / / youtu .be/1rTecr7_H6I.

[13] Beretta, G. B., "Functional color selection system.” U.S. Patent 5,311,212 (May 10 1994).

[14] Beretta, G. B., "Graphical user interface for editing a palette of colors." U.S. Statutory Invention Registration H1506 (December 5 1995).

[15] Beretta, G. B., "Meta-palette.” Xerox PARC Video (June 12 1988). http: / youtu . be / sR8Uz OQF 9VQ.

[16] Beretta, G. B., "Reference color selection system." U.S. Patent 5,254,978 (October 19 1993).

[17] Moroney, N. M., "Unconstrained web-based color naming experiment," in [Color Imaging VIII: Processing, Hardcopy, and Applications ], Eschbach, R. and Marcu, G. G., eds., 5008, 36-46, SPIE (January 2003).

[18] Mylonas, D., MacDonald, L., and Wuerger, S., "Towards an online color naming model," in [Eighteenth Color Imaging Conference: Color Science and Engineering Systems, Technologies, and Applications], Imai, F. H. and Langendijk, E., eds., 18, 140-144, IS\&T, Springfield, VA, San Antonio, Texas (November 2010).

[19] Munroe, R. and Ellis, E., "xkcd: the blag of the webcomic." Web Page (March 2010). http://blog.xkcd. $\mathrm{com} / 2010 / 03 /$.

[20] Moroney, N. M. and Beretta, G. B., "Validating large-scale lexical color resources," in [Interaction of Colour and Light in the Arts and Sciences], Schindler, V. M. and Funt, B., eds., Midterm Meeting of the International Colour Association (AIC) (June 2011).

[21] Zuffi, S., Scala, P., Brambilla, C., and Beretta, G. B., "Web-based versus controlled environment psychophysics experiments," in [Image Quality and System Performance IV], Cui, L. C. and Miyake, Y., eds., 6494, 49407-49407, SPIE (January 2007).

[22] Berlin, B. and Kay, P., [Basic Color Terms: Their Universality and Evolution], University of California Press, Berkeley (1969).

[23] O’Donovan, P., Agarwala, A., and Hertzmann, A., "Color compatibility from large datasets," ACM Trans. Graph. 30, 63:1-63:12 (August 2011). http://doi.acm.org/10.1145/2010324.1964958.

[24] Lavendel, L. and Kohler, T., "The story of a color advisor," in [Sixth Color Imaging Conference: Color Science, Systems and Applications], Süsstrunk, S. and Lakatos, A., eds., 6, 228-229, IS\&T and SID, The Society for Imaging Science and Technology, Scottsdale (Arizona) (November 1998).

[25] Moroney, N., Obrador, P., and Beretta, G. B., "Lexical image processing," in [Sixteenth Color Imaging Conference: Color Science and Engineering Systems, Technologies, and Applications], Larimer, J. and Moroney, N., eds., 16, 268-273, The Society for Imaging Science and Technology Society for Information Display (November 10-14 2008).

[26] Kelly, K. L. and Judd, D. B., [The ISCC-NBS Method of Designating Colors and a Dictionary of Color Names], vol. 553, National Bureau of Standards, Washington (November 1955).

[27] Nemcsics, A., [Colour Dynamics: Environmental Colour Design], Akadémiai Kiadó, Budapest (1993).

[28] Tominaga, S., Ono, A., and Horiuchi, T., "Investigation and analysis of color terms in modern Japanese," Color Imaging XV: Displaying, Processing, Hardcopy, and Applications 7528(1), 752804, SPIE (2010). http://dx. doi.org/10.1117/12.838884.

[29] Skaff, S., Marchesotti, L., Csurka, G., and Sauders, C., "A study on perceptually coherent distance measures for color schemes," in [Nineteeth Color Imaging Conference: Color Science and Engineering Systems, Technologies, and Applications ], Woolfe, G. and Ferweda, J., eds., 247-252 (November 2011). 